\title{
Invertible Relations on Polytopes
}

\author{
G. T. Sallee \\ Department of Mathematics, University of California, Davis, CA 95616, USA \\ Communicated by G.CC. Rota
}

\begin{abstract}
If $g$ and $h$ are any nonzero functions on the class of convex polytopes then $\alpha\left(F^{i}, F^{j}\right)=g\left(F^{i}\right) / h\left(F^{j}\right)$ is a valuation whose inverse is $\omega\left(F^{i}, F^{j}\right)=$ $(-1)^{j-i} h\left(F^{i}\right) / g\left(F^{j}\right)$. This is proved and a smaller class of valuations are characterized: those $\alpha\left(F^{i}, F^{j}\right)$ which depend only on $i$ and $j$ and which have inverses of the same form.
\end{abstract}

\section{Introduction}

Let $\mathscr{P}^{d}$ be the class of all $d$-dimensional convex polytopes in $E^{d}, d$-dimensional Euclidean space (see Grünbaum [1] for definitions and basic facts). A few years ago, McMullen [3] demonstrated an interesting method of constructing invertible functions on faces of a polytope $P$ : if $\varphi$ is any function defined on the faces of $P$, and $F^{j}$ denotes a $j$-dimensional face of $P$, we may define

$$
\psi\left(F^{j}\right)=\sum(-1)^{j-i} \beta\left(F^{i}, F^{j}\right) \varphi\left(F^{i}\right)
$$

where the sum extends over all nonempty faces $F^{i} \subseteq F^{j}$ and $\beta\left(F^{i}, F^{j}\right)$ denotes the internal angle of $F^{i}$ at $F^{j}$ normalized so that the total measure is 1 . This method is of interest largely because $\varphi$ may be recovered from $\psi$ in much the same way (see [2])

$$
\varphi\left(F^{i}\right)=\sum \gamma\left(F^{i}, F^{j}\right) \psi\left(F^{i}\right)
$$

where the sum is again taken over all nonempty faces $F^{i} \subseteq F^{j}$ and $\gamma\left(F^{i}, F^{j}\right)$ denotes the (normalized) external angle of $F^{i}$ at $F^{j}$.

The underlying ideas appear more slearly when viewed (as in [2]) in terms of Rota's Incidence Algebra $A(P)$ defined on the lattice of faces of $P$ where the lattice ordering is defined by set inclusion [4]. Here, an element $\Theta \in A(P)$ is 
defined on pairs of faces of $P$ where $\Theta\left(F^{i}, F^{j}\right)=0$ unless $F^{i} \subseteq F^{j}$. Multiplication in the algebra is defined by $\Theta \circ \tau\left(F^{i}, F^{j}\right)=\sum \Theta\left(F^{i}, F^{k}\right) \tau\left(F^{k}, F^{j}\right)$ where the terms of the sum vanish unless $F^{i} \subseteq F^{k} \subseteq F^{j}$. The identity element is $\iota\left(F^{i}, F^{j}\right)=1$ iff $F^{i}=F^{j}$.

For any function $\varphi$ defined on the faces of $P$, we may also define an element $\varphi_{1}$ of $A(P)$ by $\varphi_{1}\left(F^{i}, F^{j}\right)=\varphi\left(F^{j}\right)$. If we also write $\Theta^{*}\left(F^{i}, F^{j}\right)=(-1)^{j-i} \Theta\left(F^{i}, F^{j}\right)$ for $\Theta \in A(P)$, then McMullen's result appears as:

$$
\psi_{1}=\varphi_{1} \circ \beta^{*} \text { and } \varphi_{1}=\psi_{1} \circ \gamma
$$

This in turn depends on his identity $\beta^{*} \circ \gamma=\iota$.

The question naturally arises as to what other functions $\alpha$ and $\omega$ in $A(P)$ could play the same roles as $\beta^{*}$ and $\gamma$ ? Or for what $\alpha$ and $\omega$ do we have $\alpha \circ \omega=\imath$ ? In general, for any nonzero $\alpha$ you may construct a suitable inverse, $\omega$, but there will be little intuitive relationship between the two functions.

However, in the special case that $\alpha$ and $\omega$ depend solely on the dimensions of the faces involved-that is, when $\alpha\left(F^{i}, F^{j}\right)\left[\omega\left(F^{i}, F^{j}\right)\right]$ may be written as $\alpha(i, j)[\omega(i, j)$, respectively] - the answer is quite nice. The description arises from the observation that one large class of functions in $A(P)$ is easy to invert. These are the functions described in Theorem 1 which are of the form $G(i) / H(j)$, where $G$ and $H$ are arbitrary functions. It turns out that very little additional freedom is allowed in the most general case: there is some real number $\lambda$ and $G(i) / H(j)$ is multiplied by powers of $(1 \pm \lambda)$. The exact description of these more general functions is given in Theorem 2. Showing that they, in fact, characterize the functions of the required type constitutes the bulk of this paper.

Theorem 1. Suppose that an element of $A(P)$ is defined by $\alpha\left(F^{i}, F^{j}\right)=\alpha(i, j)=$ $G(i) / H(j)$ where $G$ and $H$ are nonzero functions defined on the integers. Then $\alpha^{-1}\left(F^{i}, F^{j}\right)=\omega(i, j)=(-1)^{j-i} H(i) / G(j)$.

Theorem 2. Let $\alpha$ be an element of $A(P)$ such that $\alpha\left(F^{i}, F^{j}\right)=\alpha(i, j) \neq 0$ and such that $\alpha^{-1}\left(F^{i}, F^{j}\right)=\omega(i, j)$. Then there exist nonzero functions $G$ and $H$ defined on the integers and a constant $\lambda$ such that $\alpha(i, i)=1 / \omega(i, i)=G(i) / H(i)$ and, for $i<j$ :

$$
\begin{aligned}
& \alpha(i, j)=G(i) / H(j) z(j-1), \\
& \omega(i, j)=(-1)^{j-i} H(i) / G(j) z(i),
\end{aligned}
$$

where

$$
z(k)= \begin{cases}1+\lambda & \text { if } k \text { is even } \\ 1-\lambda & \text { if } k \text { is odd }\end{cases}
$$




\section{Proof of Theorem 1}

We will actually establish a more general result which contains Theorem 1 as a special case:

Suppose that an element of $A(P)$ is $\alpha\left(F^{\prime}, F^{\prime}\right)=G\left(F^{\prime}\right) / H\left(F^{\prime}\right)$ where $G$ and $H$ are nonzero functions defined on polytopes. Then

$$
\omega\left(F^{i}, F^{\prime}\right)=\alpha^{-1}\left(F^{\prime}, F^{\prime}\right)=(-1)^{j-1} H\left(F^{\prime}\right) / G\left(F^{\prime}\right) .
$$

Denote by $f_{k}\left(F^{i}, F^{j}\right)$ the number of $k$-faces, $F^{k}$, of a $d$-polytope $P$ such that $F^{i} \subseteq F^{k} \subseteq F^{j}$, and let $f_{k}=f_{k}(\varphi, P)$. Then there are two standard results we need $([1$, p. 137 and p. 131]).

Euler's Formula: If $F^{i} \subseteq F^{j}$ are faces of a $d$-polytope $P$, then

$$
\sum_{k=i}^{j}(-1)^{k} f_{k}\left(F^{i}, F^{j}\right)=0 \quad \text { if } i \neq j
$$

Suppose $\sum_{k=i+1}^{j-1} \lambda_{k} f_{k}\left(F^{i}, F^{\prime}\right)=\mu_{i j}$ is a linear relation which holds for all pairs of faces $F^{\prime} \subseteq F^{\prime}$ of all convex d-polytopes. Then

$$
\lambda_{i+1}=-\lambda_{i+2}=\cdots=(-1)^{j-i} \lambda_{j-1} \text { and } \mu_{i j}=\lambda_{i+1}\left[1+(-1)^{j-i}\right]
$$

Proof of (3). Suppose $\alpha$ is given as in the statement of the theorem, and $\omega\left(F^{i}, F^{j}\right)=(-1)^{j-i} H\left(F^{i}\right) / G\left(F^{j}\right)$. We must show $\alpha \circ \omega=\iota$. If $F^{i}=F^{j}$, the asser* tion is trivial, so suppose $F^{i} \neq F^{j}$. Then

$$
\begin{aligned}
\alpha \circ \omega\left(F^{i}, F^{j}\right) & =\sum \alpha\left(F^{i}, F^{k}\right) \omega\left(F^{k}, F^{j}\right) \\
& =\sum\left(G\left(F^{i}\right) / H\left(F^{k}\right)\right)(-1)^{j-k}\left(H\left(F^{k}\right) / G\left(F^{j}\right)\right) \\
& =\left(-1, G\left(F^{i}\right) / G\left(F^{j}\right) \sum(-1)^{k},\right.
\end{aligned}
$$

where each sum is taken over all $k$-faces $F^{k}$ such that $F^{i} \subseteq F^{k} \subseteq F^{j}$. But (4) tells us that this last sum is 0 and the theorem is proved.

\section{Proof of Theorem 2}

The proof essentially involves showing that all the $\alpha(i, j)$ are determined by $\alpha(i, i)$ and $\alpha(i, i+1)(i \geq-1)$ and by $\alpha(-1,1)$. The function $z(k)$ then arises in a natural way from the fact that $\alpha(i, j)$ is computed from $\alpha(i-1, j-1)$ by multiplying the latter by either $1+\lambda$ or $1-\lambda$.

We first define some auxiliary functions $g$ and $h$. Set $h(-1)=1$. Then define $h(j+1)=h(j) \alpha(j, j) / \alpha(j, j+1)$ and $g(j)=g(j) \alpha(j, j)$. Then by definition $\alpha(i, i)=g(i) / h(i)$ and $\alpha(i, i+1)=g(i) / h(i+1)$. 
At this point it is possible to define $\lambda$. Let $\alpha(-1,1)=(1-\lambda) g(-1) / h(1)$ and let $z(n)$ be defined as in the statement of Theorem 2 . Finally, we define the functions used in our theorem:

$$
G(i)=g(i) z(i-1) z(i-2) \cdots z(-2),
$$

and

$$
H(j)=h(j) z(j-1) z(j-2) \cdots z(-2) .
$$

Note that $G(i) / H(i)=g(i) / h(i)=\alpha(i, i)$, and

$$
G(i) / H(i+1) z(i)=g(i) / h(i+1)=\alpha(i, i+1) .
$$

Let $\omega$ be the inverse function to $\alpha$; that is, $\alpha \circ \omega=\imath$ in $A$. Writing out this multiplication explicitly shows that:

$$
\begin{aligned}
\alpha(i, i) \omega(i, i) & =1 \text { for all } i . \\
\sum_{k=i}^{j} \alpha(i, k) \omega(k, j) f_{k}\left(F^{i}, F^{j}\right) & =0 \quad \text { if } F^{i} \subseteq F^{j} \text { and } i \neq j
\end{aligned}
$$

Now by using (5) in conjunction with (7), we get two further sets of equations for all $i<j$. The designations of the equations are at the left.

$$
\begin{array}{ll}
(i, j, k) & \alpha(i, k) \omega(k, j)=-\alpha(i, k+1) \omega(k+1, j), \quad i+1 \leq k \leq j-2, \\
(i, j, *) & \alpha(i, i) \omega(i, j)+\alpha(i, j) \omega(j, j)=-\alpha(i, i+1) \omega(i+1, j)\left(1+(-1)^{j-i}\right) .
\end{array}
$$

Now from (6), we get $\omega(i, i)=1 / \alpha(i, i)=H(i) / G(i)$. Then since $\alpha \circ \omega\left(F^{i}, F^{i+1}\right)=\alpha(i, i) \omega(i, i+1)+\alpha(i, i+1) \omega(i+1, i+1)=0$, we see $\omega(i, i+1)$ $=-H(i) / G(i+1) z(i)$. Thus $\omega(i, j)$ is also of the proper form for $j=i, i+1$.

Now we combine equations $(i, i+2, *)$ and $(i-1, i+2, i)$ to show, after a little algebra, that

$$
\begin{aligned}
\alpha(i, i+2)= & \omega(i+1, i+2) \alpha(i+2, i+2) \\
& \times\left[-2 \alpha(i, i+1)+\frac{\alpha(i, i)}{\alpha(i-1, i)} \alpha(i-1, i+1)\right]
\end{aligned}
$$

But since we know most of the terms, this expression simplifies to:

$$
\alpha(i, i+2)=\frac{G(i)}{H(i+2) z(i) z(i-1)}\left[2-\frac{H(i+1) \alpha(i-1, i+1)}{G(i-1) z(i-1)}\right] .
$$

Using the fact that $\alpha(-1,1)=F(-1) / H(1) z(0)$ and noting that $2-z(i)=z(i+1)$, (9) may be employed inductively to get, for all $i \geq-1$ :

$$
\alpha(i, i+2)=G(i) / H(i+2) z(i+1) .
$$

Using (10) in conjunction with equation $(i-1, i+2, i)$ we find $\omega(i, i+2)=$ $H(i) / G(i+2) z(i)$. 
The rest is easy to do by induction on $n=j-i$. So far the theorem has been proved for both $\alpha(i, j)$ and $\omega(i, j)$ when $j-i=0,1,2$. Assume it is known if $j-i \leq n$. Then from equation $(i, i+n+2, i+n)$ we have

$$
\alpha(i, i+n+1)=-\alpha(i, i+n) \omega(i+n, i+n+2) / \omega(i+n+1, i+n+2) .
$$

Using our induction hypothesis this becomes

$$
\begin{aligned}
\alpha(i, i+n+1)= & \left\{\frac{-G(i)}{H(i+n)} z(i+n-1)\right\} \\
& \times\left\{\frac{H(i+n)}{G(i+n+2)} z(i+n)\right\}\left\{-\frac{G(i+n+2)}{H(i+n+1)} z(i+n+1)\right\} \\
= & \frac{G(i)}{H(i+n+1)} z(i+n),
\end{aligned}
$$

since $z(k)=z(k+2)$ for all integers $k$. We may verify the induction step for $\omega(i, i+n+1)$ in the same way by using equation $(i-2, i+n+1, i)$.

\section{Remarks}

1. These results generalize the Euler relation which is the special case arising when $\alpha(i, i)=-\alpha(i, i+1)=\alpha(-1,1)=1$.

2. The result stated in (3) can be extended even further to the case where $\alpha\left(F^{i}, F^{j}\right)=G\left(F^{i}\right) / H\left(F^{j}\right) z(j-1)$, and $z$ is the function described in Theorem 2.

3. The set of values $\alpha(i, i), \alpha(i, i+1), i \geq 1$, and $\alpha(-1,1)$ will generate an element of the incidence algebra whenever $\alpha(i, i) \neq 0$. However, it is much more difficult to write down the values of the $\alpha(i, j)$ explicitly if some of the $\alpha(i, i+1)=$ 0 . When this happens, many different cases arise of patterns of $\alpha(i, j)$ which are forced to be zero. The only easily described case occurs when $\alpha(-1,1)=0$. Then $\lambda=1$ and the results of Theorem 2 hold when recast in a form using the auxiliary functions $g$ and $h$.

\section{Acknowledgment}

I am grateful to the referee for pointing out a great improvement in the notation used in the statement of Theorem 2.

\section{References}

1. B. Grünbaum, Convex Polytopes, Wiley, London, 1967.

2. P. McMullen, Non-linear angle-sum relations for polyhedral cones and polytopes, Math. Proc. Cambridge Philos. Soc. 78 (1975), 247-261.

3. P. McMullen, Valuations and Euler-type relations on certain classes of convex polytopes, Proc. London Math. Soc. (3) 35 (1977), 113-135.

4. G. C. Rota, On the foundations of combinatorial theory, (1). Theory of Möbius functions, $Z$. Wahrsch. Verw. Gebiete 2 (1964), 340-368.

Received August 20, 1985, and in revised form June 2, 1986. 